\title{
Interleukin 8 in progression of hormone-dependent early breast cancer
}

\author{
Jelena Milovanović* (i), Nataša Todorović-Raković, Tijana Vujasinović and Zaki Abu Rabi \\ Department of Experimental Oncology, Institute of Oncology and Radiology of Serbia, Belgrade, Serbia \\ *Corresponding author (Email, jelena.mil10@gmail.com)
}

\begin{abstract}
The only way to perceive the real clinical course of disease and the prognostic significance of potential biomarkers is follow-up of patients who did not receive any kind of adjuvant therapy. Many studies have confirmed high levels of interleukin 8 (IL8) in HER2-enriched and basal-like (ER-) primary breast tumours, but less is known about the significance of IL8 in hormone-dependent breast cancer. The aim of this study was to evaluate the prognostic significance of IL8 and clinicopathological parameters in hormone-dependent breast cancer, and to examine possible associations between them that might imply possible biological dependence. The study included 91 early-stage breast cancer patients with detectable levels of hormone receptors $(E R>0, P R>0)$. None of the patients received adjuvant therapy according to valid protocol at that time. HER2 status was determined on paraffin-embedded tumour tissue sections by CISH. IL8 levels were determined by ELISA in cytosol tumour extracts of 65 patients with long-term follow-up (144 months). Nonparametric statistical tests were used for data analyses. Patients with low IL8 levels $(\mathrm{M}<88.8 \mathrm{pg} / \mathrm{mg}$ ) had significantly longer relapse-free survival (RFS) compared to patients with high IL8 levels $(\mathrm{M} \geq 88.82 \mathrm{pg} / \mathrm{mg}$ ) (Log rank test, $\mathrm{p}=0.002$ ). Patients with ERhighIL8low phenotype had significantly longer RFS compared to those with ERhighIL8high and ERlowIL8high phenotypes ( $\mathrm{p}=0.04$ and $\mathrm{p}=0.02$, respectively); patients with PRlowIL8low phenotype had significantly longer RFS compared to those with PRlowIL8high and PRhighIL8high phenotypes ( $\mathrm{p}=0.003$ and $\mathrm{p}=0.02$, respectively); patients with HER2-IL8low phenotype had significantly longer RFS compared to those with HER2-IL8high and HER2+IL8high phenotypes ( $\mathrm{p}=0.01$ and $\mathrm{p}=0.02$, respectively). Our results indicate significant contribution of IL8 on survival of hormone-dependent early-stage breast cancer patients and association with established parameters such as ER/PR and HER2.
\end{abstract}

[Milovanović J, Todorović-Raković N, Vujasinović T and Abu Rabi Z 2017 Interleukin 8 in progression of hormone-dependent early breast cancer. J. Biosci. 42 265-274]

\section{Background}

Breast cancer is hormone-dependent disease with great genetical, pathological and clinical heterogeneity. Hormone receptors (ER and PR) together with human epidermal growth factor receptor 2 (HER2) are the main determinants of breast cancer and still the most important established biomarkers. The only way to perceive the real clinical course of disease and the prognostic significance of potential biomarkers is follow-up of patients who did not receive any kind of adjuvant (postoperative) therapy and had only surgical intervention. Very little data is available regarding this issue because some kind of adjuvant therapy is given to all breast cancer patients nowadays.

Interleukin 8 (IL8) has been intensively examined in recent years as potential prognostic biomarker in different types of human cancers. IL8 is an inflammatory cytokine that belongs to the class of CXC chemokines, a potent chemoattractant and activator of neutrophils, monocytes and other immune cells. Elevated expression of IL8 has been characterized in many different chronic inflammatory conditions and different types of human cancers (reviewed in Qazi et al. 2011). Literature have indicated that IL8 could contribute to tumour growth and progression directly, by

Keywords. Breast cancer; HER2; hormone receptor; interleukin 8 
Table 1. Patient's clinicopathological parameters at the time of primary diagnosis

\begin{tabular}{|c|c|c|}
\hline Clinicopathological parameters & $\mathrm{n}$ & $\%$ \\
\hline \multicolumn{3}{|l|}{ Age (years) } \\
\hline$<62$ (median) & 44 & 48.4 \\
\hline$\geq 62$ & 47 & 51.6 \\
\hline NA & 0 & 0 \\
\hline \multicolumn{3}{|l|}{ Menopausal status } \\
\hline Premenopausal & 0 & 0 \\
\hline Postmenopausal & 91 & 100 \\
\hline NA & 0 & 0 \\
\hline \multicolumn{3}{|l|}{ Tumour size (cm) } \\
\hline $\mathrm{T}<2$ & 49 & 53.8 \\
\hline $\mathrm{T} \geq 2$ & 40 & 44.0 \\
\hline NA & 2 & 2.2 \\
\hline \multicolumn{3}{|l|}{ Nodal status } \\
\hline N0 & 91 & 100 \\
\hline $\mathrm{N}+$ & 0 & 0 \\
\hline NA & 0 & 0 \\
\hline \multicolumn{3}{|l|}{ Histological type } \\
\hline IDC & 36 & 39.5 \\
\hline ILC & 27 & 29.7 \\
\hline Other histologies & 26 & 28.6 \\
\hline NA & 2 & 2.2 \\
\hline \multicolumn{3}{|l|}{ Tumour grade } \\
\hline G1 & 9 & 9.9 \\
\hline G2 & 80 & 87.9 \\
\hline G3 & 1 & 1.1 \\
\hline NA & 1 & 1.1 \\
\hline \multicolumn{3}{|l|}{ Estrogen receptor status } \\
\hline ERlow & 20 & 22.0 \\
\hline ERhigh & 71 & 78.0 \\
\hline NA & 0 & 0 \\
\hline \multicolumn{3}{|l|}{ Progesterone receptor status } \\
\hline PRlow & 70 & 76.9 \\
\hline PRhigh & 21 & 23.1 \\
\hline NA & 0 & 0 \\
\hline \multicolumn{3}{|l|}{ HER2 status } \\
\hline HER2- & 65 & 71.4 \\
\hline HER2+ & 20 & 22.0 \\
\hline NA & 6 & 6.6 \\
\hline \multicolumn{3}{|l|}{ Receptor status } \\
\hline ERlowPRlow & 20 & 22.0 \\
\hline ERlowPRhigh & 0 & 0 \\
\hline ERhighPRlow & 50 & 54.9 \\
\hline ERhighPRhigh & 21 & 23.1 \\
\hline NA & 0 & 0 \\
\hline \multicolumn{3}{|l|}{ Receptor status } \\
\hline ERlowPRlowHER2- & 10 & 11.0 \\
\hline ERhighPRhighHER2+ & 2 & 2.2 \\
\hline NA & 6 & 6.6 \\
\hline
\end{tabular}

n, number of patients; IDC, invasive ductal carcinoma; ILC, invasive lobular carcinoma; ERlow, ER $<10 \mathrm{fmol} / \mathrm{mg}$; ERhigh, ER $\geq 10$ fmol/mg; PRlow, PR $<20 \mathrm{fmol} / \mathrm{mg}$; PRhigh, $P R \geq 20 \mathrm{fmol} / \mathrm{mg}$; HER2-, HER2 gene not amplified; HER2+, HER2 gene amplified; NA, data not available. acting as mitogenic and potent angiogenic factor, as well as indirectly, by chemotactic infiltration of immune cells that secrete by themselves growth and angiogenic factors (reviewed in Xie 2001). Moreover, recent studies have supported the involvement of IL8 signaling in regulation of stem cells activity, which is considered to be responsible for tumour initiation, maintenance, metastases and disease recurrence (Singh et al. 2013). Many studies have indicated that there are high levels of IL8 in basal-like (ER-) and HER2-enriched (HER2+) primary breast tumours, two subtypes characterized by a particularly poor prognosis (Aceto et al. 2012), but less is known about the role and the significance of IL8 in hormone-dependent breast cancer.

The aims of the study were to evaluate the prognostic significance of interleukin 8 and clinicopathological parameters in hormone-dependent breast cancer, and to examine possible associations between them that might imply possible biological dependence.

\section{Materials and methods}

The study included 91 early-stage breast cancer patients (clinical stadium I/II) with known clinicopathological parameters. All patients were postmenopausal and had detectable levels of hormone receptors $(\mathrm{ER}>0, \mathrm{PR}>0)$ that should indicate hormone-dependent breast cancer. All patients underwent surgical removal of their primary tumour at the Institute of Oncology and Radiology of Serbia. After surgery, histological specimens were examined and classified according to the criteria of AJCC/UICC (American Joint Committee on Cancer/Union International Contre le Cancer) for TNM stage and histological type, and part of the tumour was frozen. Age and menopausal status, regional lymph node status $(\mathrm{N})$, tumour size $(\mathrm{T})$, tumour grade $(\mathrm{G})$ and histological type were obtained after the Institutional Review Board approval. None of the patients received adjuvant therapy according to valid protocol at that time, due to favorable clinicopathological parameters, preferentially N0 status and absence of G3 tumour grade. The course of disease was followed from surgical intervention till relapse, so-called, relapse-free survival (RFS).

HER2 status (absence or presence of gene amplification) was determined on formalin-fixed paraffin-embedded tumour tissue sections by chromogenic in situ hybridization (CISH), according to the manufacturer's instructions (Invitrogen SPOT-Light HER2 CISH Kit, USA). Hybridization results were evaluated in $40 \times$ and $100 \times$ magnification (Olympus BX51 light microscope). One to 5 gene copies per nucleus were defined as no amplification, while more than 6 gene copies per nucleus or large gene copy clusters in more than $50 \%$ of tumour cells defined amplification. 
Table 2. Survival analyses for subgroups of patients formed according to available clinicopathological parameters

\begin{tabular}{|c|c|c|c|c|c|c|}
\hline $\begin{array}{l}\text { Clinicopathological } \\
\text { parameters }\end{array}$ & $\begin{array}{l}\text { Number of } \\
\text { patients }\end{array}$ & $\begin{array}{l}\text { Number of } \\
\text { relapses }\end{array}$ & Relapses (\%) & RFS (months) & Median RFS (months) & $\begin{array}{l}\text { p-Value } \\
\text { (Log Rank) }\end{array}$ \\
\hline \multicolumn{7}{|l|}{ Age (years) } \\
\hline$<62$ (median) & 44 & 12 & 28.6 & $4-161$ & 95 & 0.5 \\
\hline$\geq 62$ & 47 & 11 & 23.9 & $3-165$ & 127 & \\
\hline NA & 0 & & & & & \\
\hline \multicolumn{7}{|l|}{ Tumour size (cm) } \\
\hline $\mathrm{T}<2$ & 49 & 8 & 16.3 & $3-161$ & 144 & 0.03 \\
\hline $\mathrm{T} \geq 2$ & 40 & 14 & 35 & $3-165$ & 95 & \\
\hline NA & 2 & & & & & \\
\hline \multicolumn{7}{|l|}{ Histological type } \\
\hline IDC & 36 & 5 & 13.9 & $6-165$ & 145 & 0.01 \\
\hline ILC & 27 & 11 & 40.7 & $3-158$ & 69 & \\
\hline NA & 2 & & & & & \\
\hline \multicolumn{7}{|l|}{ Tumour grade } \\
\hline G1 & 9 & 1 & 11.1 & $24-158$ & 145 & 0.3 \\
\hline G2 & 80 & 22 & 27.5 & $3-165$ & 103.5 & \\
\hline NA & 1 & & & & & \\
\hline \multicolumn{7}{|l|}{ ER status } \\
\hline ERlow & 20 & 5 & 25 & $3-162$ & 65 & 0.6 \\
\hline ERhigh & 71 & 18 & 25.3 & $3-165$ & 108 & \\
\hline NA & 0 & & & & & \\
\hline \multicolumn{7}{|l|}{ PR status } \\
\hline PRlow & 70 & 19 & 27.1 & $3-165$ & 106.5 & 0.5 \\
\hline PRhigh & 21 & 4 & 19 & $4-165$ & 97 & \\
\hline NA & 0 & & & & & \\
\hline \multicolumn{7}{|l|}{ Receptor status } \\
\hline ERlowPRlow & 20 & 5 & 25 & $3-162$ & 65 & 0.5 \\
\hline ERhighPRhigh & 21 & 4 & 19 & $4-165$ & 97 & \\
\hline NA & 0 & & & & & \\
\hline \multicolumn{7}{|l|}{ HER2 status } \\
\hline HER2-- & 65 & 16 & 24.6 & $3-165$ & 114 & 0.8 \\
\hline HER2+ & 20 & 5 & 25 & $10-155$ & 101 & \\
\hline NA & 6 & & & & & \\
\hline
\end{tabular}

IDC, invasive ductal carcinoma; ILC, invasive lobular carcinoma; ERlow, ER $<10 \mathrm{fmol} / \mathrm{mg}$; ERhigh, ER $\geq 10$ fmol/mg; PRlow, PR $<20$ fmol/mg; PRhigh, PR $\geq 20$ fmol/mg; HER2-, HER2 gene not amplified; HER2+, HER2 gene amplified; NA, data not available.

Table 3. Survival analysis for subgroups of patients formed according to the median IL8 level

\begin{tabular}{lcccccc}
\hline Biomarker & Number of patients* & Number of relapses & Relapses (\%) & RFS (months) & Median RFS (months) & $\begin{array}{l}\text { p-Value } \\
\text { (Log Rank) }\end{array}$ \\
\hline IL8 & & & & & 150 & 0.002 \\
IL8low & 33 & 5 & 15.1 & $19-161$ & 96 \\
IL8high & 32 & 14 & 43.7 & $3-165$ & \\
\hline
\end{tabular}

*IL8 levels were measured in cytosol tumour extracts of 65 patients with long-term follow-up (144 months).

IL8low, IL8<88.82 pg/mg; IL8high, IL $8 \geq 88.82 \mathrm{pg} / \mathrm{mg}$. 


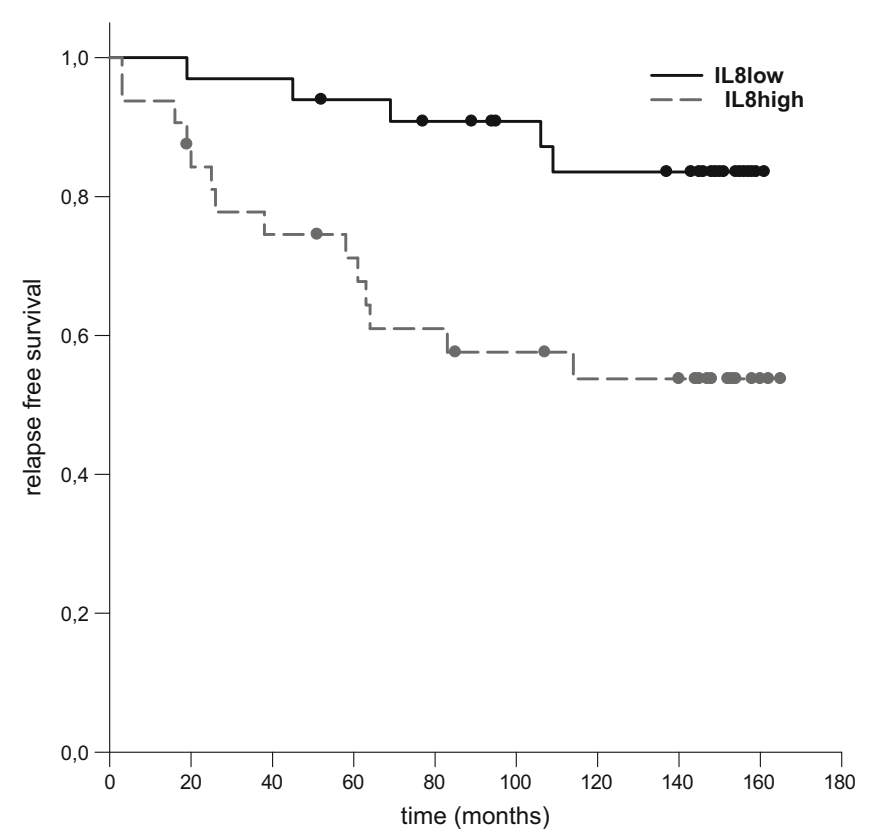

Figure 1. Survival analysis for subgroups of patients formed according to the median IL8 level (M=88.82 pg/mg).

Cytosol tumour extracts were prepared from frozen tumours by a following workflow: homogenization in $5 \mathrm{mM}$ phosphate buffer $\mathrm{pH}$ 7.4-7.7 containing $20 \%$ glycerol, $1 \mathrm{mM}$ monothioglycerol and $1.5 \mathrm{mM}$ EDTA, centrifugation at $800-1000 \mathrm{~g}$ for $30 \mathrm{~min}$, followed by ultracentrifugation at
$100000 \mathrm{~g}$ for $60 \mathrm{~min}$ (ultracentrifuge Beckman L5-50). The whole procedure was performed at $+4^{\circ} \mathrm{C}$. Tumour extract protein concentrations were assayed by the Lowry method. Hormone receptor concentrations were determined by the standard ligand-binding assay. ER levels $<10 \mathrm{fmol} / \mathrm{mg}$ and PR levels $<20 \mathrm{fmol} / \mathrm{mg}$ was considered as low. ER levels $\geq 10 \mathrm{fmol} / \mathrm{mg}$ and PR levels $\geq 20 \mathrm{fmol} / \mathrm{mg}$ was considered as high.

IL8 concentrations were determined by ELISA in cytosol tumour extracts of 65 patients with long-term follow-up (144 months), according to the manufacturer's instructions (RayBio ${ }^{\circ}$ Human IL8 ELISA Kit, USA). The assay employs an antibody specific for human IL8 coated on a 96-well plate. IL8 present in the sample is bound to the well by immobilized antibody and colour develops in proportion to the amount of IL8 bound. The median IL8 value was used as cut off value.

\subsection{Statistics}

Survival curves for RFS were constructed according to the Kaplan-Meier method and compared with the log-rank test. Associations between parameters were analysed by the Chisquare test. The Mann-Whitney rank sum test was used to examine the distribution of quantitative IL8 levels between different subgroups of patients according to clinicopathological parameters. The correlations between steroid receptors and IL8 were analysed by the Spearman's rank order correlation test. A p-value less than 0.05 were considered as statistically significant.

Table 4. Survival analyses for subgroups of patients formed according to ER status and the median IL8 level

\begin{tabular}{|c|c|c|c|c|c|c|}
\hline Phenotype & Number of patients & Number of relapses & Relapses (\%) & RFS (months) & Median RFS (months) & $\begin{array}{l}\text { p-Value } \\
\text { (Log Rank) }\end{array}$ \\
\hline ERlowIL81ow & 5 & 0 & 0 & $52-159$ & 148 & \multirow[t]{2}{*}{0.07} \\
\hline ERlowIL8high & 9 & 5 & 55.5 & $3-165$ & 114 & \\
\hline ERhighIL8low & 28 & 5 & 17.9 & $19-161$ & 150 & \multirow[t]{2}{*}{0.04} \\
\hline ERhighIL8high & 23 & 9 & 39.1 & $3-165$ & 85 & \\
\hline ERlowIL8low & 5 & 0 & 0 & $52-159$ & 148 & \multirow[t]{2}{*}{0.3} \\
\hline ERhighIL8low & 28 & 5 & 17.9 & $19-161$ & 150 & \\
\hline ERlowIL8low & 5 & 0 & 0 & $52-159$ & 148 & \multirow[t]{2}{*}{0.1} \\
\hline ERhighIL8high & 23 & 9 & 39.1 & $3-165$ & 85 & \\
\hline ERlowIL8high & 9 & 5 & 55.5 & $3-165$ & 114 & \multirow[t]{2}{*}{0.02} \\
\hline ERhighIL8low & 28 & 5 & 17.9 & 19-161 & 150 & \\
\hline ERlowIL8high & 9 & 5 & 55.5 & $3-165$ & 114 & \multirow[t]{2}{*}{0.5} \\
\hline ERhighIL8high & 23 & 9 & 39.1 & $3-165$ & 85 & \\
\hline
\end{tabular}

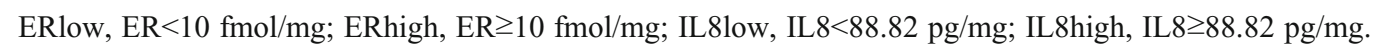



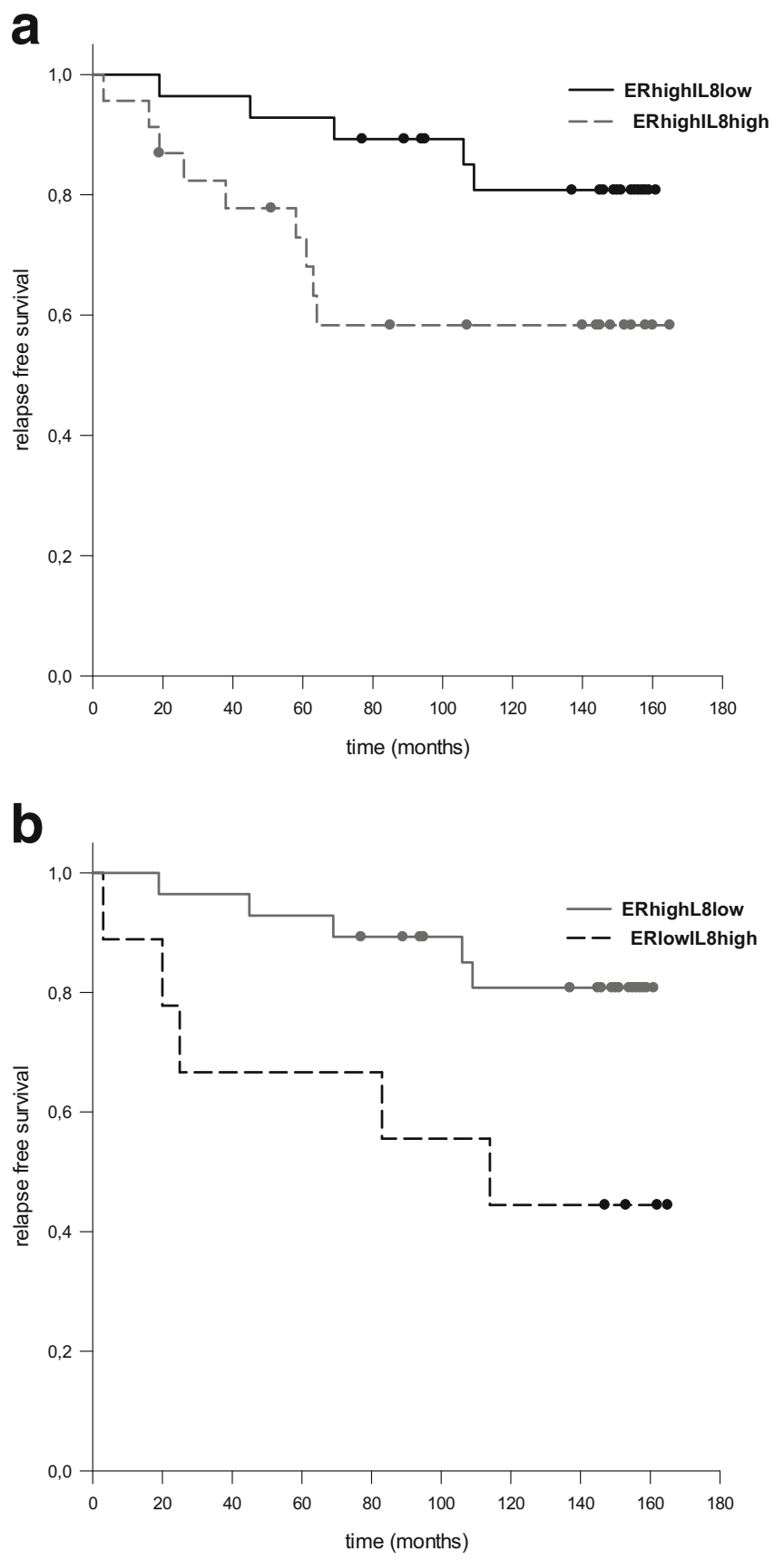

Figure 2. Survival analyses for subgroups of patients formed according to ER status and the median IL8 level (M=88.82 pg/mg).

\section{Results}

Patient's clinicopathological parameters at the time of primary diagnosis are presented in table 1 . There were no statistically significant differences in the expression of IL8 between subgroups of patients formed according to patient's age, tumour size, histological type, tumour grade and receptor status (Mann-Whitney rank sum test). There was no statistically significant correlation between ER and IL8 expression, neither between PR and IL8 expression (Spearman rank order test).

Survival analyses for subgroups of patients formed according to available clinicopathological parameters are presented in table 2 . There were no statistically significant differences in relapse-free survival (RFS) between subgroups formed according to patient's age, tumour grade and receptor status but there was a statistically significant difference in RFS between subgroups formed according to tumour size (Log rank test, $\mathrm{p}=0.03$ ) and histological type $(\mathrm{p}=0.01)$.

Survival analysis for subgroups formed according to the median IL8 value ( $M=88.82 \mathrm{pg} / \mathrm{mg}$; range $5.07-1846.32 \mathrm{pg} /$ $\mathrm{mg}$ ) is presented in table 3. There was a statistically significant difference in RFS (Log rank test, $\mathrm{p}=0.002$ ) and patients with low IL8 levels $(\mathrm{M}<88.82 \mathrm{pg} / \mathrm{mg})$ had better prognosis (figure 1).

Survival analyses for subgroups of patients formed according to ER status and the median IL8 value $(\mathrm{M}=88.82 \mathrm{pg} / \mathrm{mg})$ are presented in Table 4. In all combinations of phenotypes, patients with low IL8 levels $(\mathrm{M}<88.82 \mathrm{pg} / \mathrm{mg})$ had lower percentage of relapses and longer RFS compared to patients with high IL8 levels $(\mathrm{M} \geq 88.82 \mathrm{pg} / \mathrm{mg})$, regardless of ER status. There was a statistically significant difference in RFS between patients with ERhighIL8low and ERhighIL8high phenotypes (Log rank test, $\mathrm{p}=0.04$ ) as well as between patients with ERhighIL8low and ERlowIL8high phenotypes $(p=0.02)$. Patients with ERhighIL8low phenotype had significantly longer RFS compared to patients with ERhighIL8high phenotype (figure 2a) and patients with ERhighIL8low phenotype had significantly longer RFS compared to patients with ERlowIL8high phenotype (figure $2 b$ ).

Survival analyses for subgroups formed according to PR status and the median IL8 value ( $M=88.82 \mathrm{pg} / \mathrm{mg})$ are presented in table 5. In all combinations of phenotypes, patients with low IL8 levels had lower percentage of relapses and longer RFS compared to patients with high IL8 levels, irrespective of PR status. There was a statistically significant difference in RFS between patients with PRlowIL8low and PRlowIL8high phenotypes $(\mathrm{p}=0.003)$ as well as between patients with PRlowIL8low and PRhighIL8high phenotypes $(\mathrm{p}=0.02$ ). Patients with PRlowIL8low phenotype had significantly longer RFS compared to patients with PRlowIL8high phenotype 
Table 5. Survival analyses for subgroups of patients formed according to PR status and the median IL8 level

\begin{tabular}{|c|c|c|c|c|c|c|}
\hline Phenotype & Number of patients & Number of relapses & Relapses (\%) & RFS (months) & Median RFS (months) & $\begin{array}{l}\text { p-Value } \\
\text { (Log Rank) }\end{array}$ \\
\hline PRlowIL81ow & 24 & 2 & 8.3 & $45-161$ & 150 & 0.003 \\
\hline PRlowIL8high & 25 & 11 & 44 & $3-165$ & 107 & \\
\hline PRhighIL8low & 9 & 3 & 33.3 & $19-161$ & 109 & 0.6 \\
\hline PRhighIL 8high & 7 & 3 & 42.9 & $16-160$ & 85 & \\
\hline PRlowIL8low & 24 & 2 & 8.3 & $45-161$ & 150 & 0.07 \\
\hline PRhighIL8low & 9 & 3 & 33.3 & $19-161$ & 109 & \\
\hline PRlowIL8low & 24 & 2 & 8.3 & $45-161$ & 150 & 0.02 \\
\hline PRhighIL8high & 7 & 3 & 42.9 & $16-160$ & 85 & \\
\hline PRlowIL8high & 25 & 11 & 44 & $3-165$ & 107 & 0.5 \\
\hline PRhighIL81ow & 9 & 3 & 33.3 & $19-161$ & 109 & \\
\hline PRlowIL8high & 25 & 11 & 44 & $3-165$ & 107 & 0.8 \\
\hline PRhighIL8high & 7 & 3 & 42.9 & $16-160$ & 85 & \\
\hline
\end{tabular}

PRlow, PR<20 fmol/mg; PRhigh, PR $\geq 20$ fmol/mg; IL8low, IL8<88.82 pg/mg; IL8high, IL8 $\geq 88.82$ pg/mg.

(figure 3a) and patients with PRlowIL8low phenotype had significantly longer RFS compared to patients with PRhighIL8high phenotype (figure $3 b$ ).

Survival analyses for subgroups formed according to HER2 status and the median IL8 value ( $\mathrm{M}=88.82 \mathrm{pg} / \mathrm{mg})$ are presented in table 6 . In all combinations of phenotypes, patients with low IL8 levels had lower percentage of relapses and longer RFS compared to patients with high IL8 levels, regardless of HER2 status. There was a statistically significant difference in RFS between patients with HER2-IL8low and HER2-IL8high phenotypes ( $\mathrm{p}=0.01$ ) as well as between patients with HER2-IL8low and HER2+IL8high phenotypes $(p=0.02)$. Patients with HER2-IL8low phenotype had significantly longer RFS compared to patients with HER2IL8high phenotype (figure 4a) and patients with HER2IL8low phenotype had significantly longer RFS compared to patients with HER2+IL8high phenotype (figure 4b).

\section{Discussion}

Our results showed prognostic significance of IL8 and patients with low IL8 levels had better prognosis (figure 1). Recent literature have indicated the significance of IL8 as potential prognostic marker in different types of human cancers. Increased IL8 expression in ovarian cyst fluid, ascites, serum and tumour tissue from ovarian cancer patients correlated with poor prognosis and survival (Wang et al. 2012). Increased IL8 expression in tumour tissue from endometrial cancer patients was associated with poor survival (Mannelqvist et al. 2011). Two large clinical studies showed that increased levels of both serum IL6 and IL8 were associated with lung cancer, but only IL8 levels were in direct relation to lung cancer risk several years before diagnosis (Pine et al. 2011). Some authors indicated that IL8 could be a marker for predicting the prognosis and monitoring disease progression of pancreatic cancer patients (Chen et al. 2012) as well as colorectal cancer patients (Biasi et al. 2012). Reis et al. first indicated the prognostic relevance of IL8 mRNA expression in bladder cancer (Reis et al. 2012). Nasopharyngeal carcinoma (NPC) has the highest metastatic potential among head and neck cancers. A study on 250 patients with NPC revealed that higher IL8 expression in primary tumour tissue was an independent prognostic factor for overall survival, disease-free survival and distant metastasis-free survival (Li et al. 2012).

Literature related to the prognostic significance of IL8 in early and advanced breast cancer is not extensive. Skoog et al. showed that inflammatory breast cancer, a distinct and aggressive form of locally advanced breast cancer, was associated with high levels of IL8 (Skoog et al. 1987). According to literature, IL8 from serum and/or tumour tissue could be used to diagnose women with breast cancer and to identify patients with poor prognosis (Kozłowski et al. 2003; Benoy et al. 2004; Yao et al. 2007). Benoy et al. showed that serum IL8 had an independent prognostic significance for postrelapse survival (Benoy et al. 2004) and Yao et al. showed significance of tumour tissue IL8 for disease-free survival (Yao et al. 2007). Papaxoinis et al. showed that HER2-ECD, NO and IL8 plasma levels had prognostic value for survival of patients with metastatic breast cancer treated with weekly docetaxel (Papaxoinis et al. 2010). High pretreatment IL8 levels were of adverse prognostic value for survival and patients with high IL8 relative change had worse progression-free survival. Two large clinical studies showed that the polymorphisms in IL8 and CXCR2 genes 
a

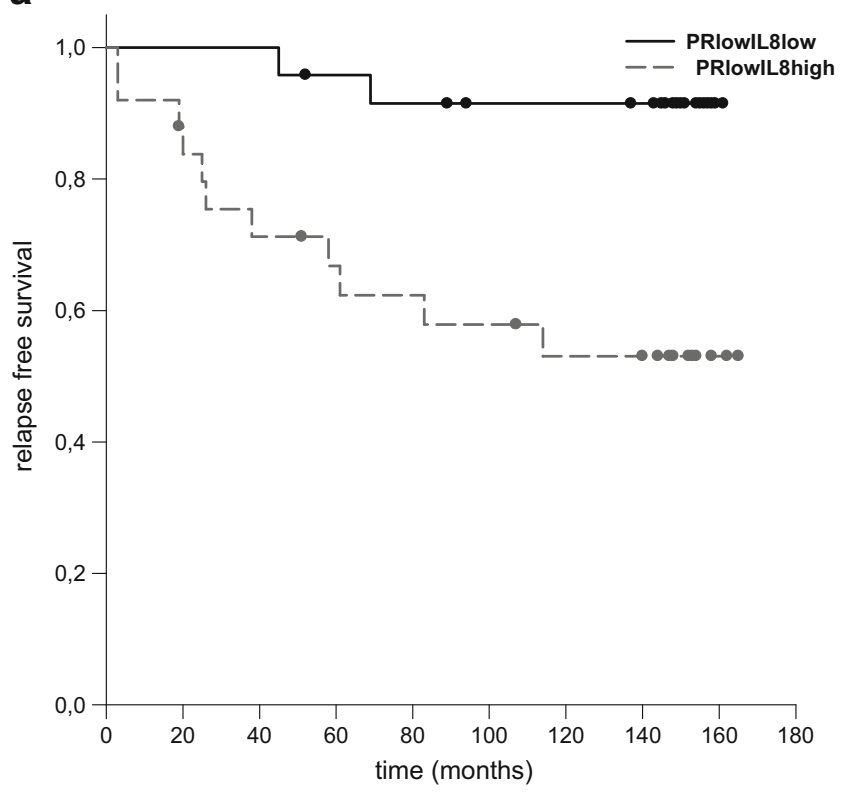

b

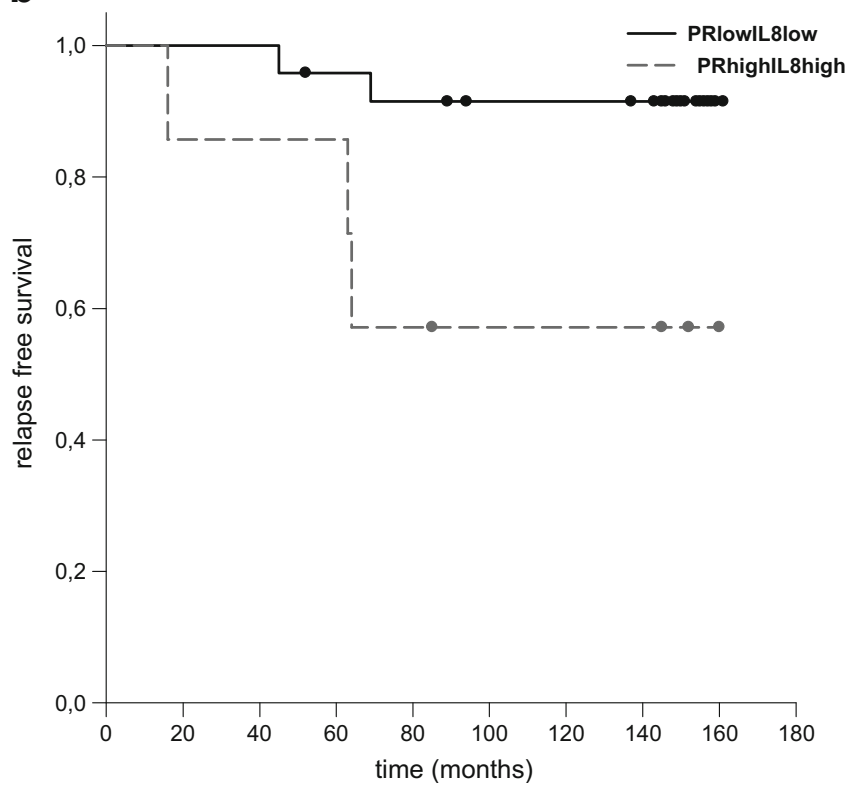

Figure 3. Survival analyses for subgroups of patients formed according to PR status and the median IL8 level ( $M=88.82 \mathrm{pg} / \mathrm{mg})$.

were associated with increased breast cancer risk and prognosis (Snoussi et al. 2010). The presence of the IL8 (-251) A allele and/or the CXCR2 (+1208) T allele showed a significant association with decreased overall survival and decreased disease-free survival. We have recently shown the prognostic relevance of IL8 in adjuvantly untreated early breast cancer patients in short-term and long-term follow-up (Milovanović et al. 2013; Abu Rabi et al. 2015).

The most important relation of IL8 is to ER and/or HER2 status, since receptor status is still the main determinant of breast cancer. According to literature, IL8 is highly expressed in ER- breast cancers and it is also highly expressed in HER2-enriched breast cancers (Freund et al. 2003; Yao et al. 2007; Aceto et al. 2012). Moreover, IL8 expression is inversely related to ER status in breast cancer cells (Freund et al. 2003; Aceto et al. 2012). It is postulated that the net effect of IL8 action in breast cancer is the result of a delicate balance between ER inhibition and HER2 induction of IL8 expression and activity (reviewed in Todorović-Raković and Milovanović 2013). In our study receptor status did not have prognostic significance (table 2), although available literature have implied that hormone receptor-positive and/or HER2negative status is generally associated with better prognosis (Clark et al. 1983; Slamon et al. 1987; Dunnwald et al. 2007).

According to our results, IL8 seems to be more reliable prognostic marker than receptor status in early-stage breast cancer. When comparing RFS of patients stratified in different phenotypes according to receptor status and the median IL8 value (tables 4, 5 and 6), in all combinations of phenotypes patients with low IL8 levels had lower percentage of relapses and longer RFS compared to patients with high IL8 levels, irrespective of ER/PR/ HER2 status.

When patients were stratified according to ER status and the median IL8 level (table 4), IL8 expression had no influence on survival of ERlow subgroup, but had significant influence on survival of ERhigh subgroup. Patients with ERhighIL8low phenotype had almost twice longer median RFS (150 months) compared to patients with ERhighIL8high phenotype (85 months) and the number of relapses in ERhighIL8low subgroup was $18 \%$ versus $39 \%$ in ERhighIL8high subgroup. This implies the significant role of IL8 in progression of hormone-dependent breast cancer, in contrast to the previously accepted hypothesis that IL8 expression is important feature that exclusively influences the course of disease of ER- breast cancer. Furthermore, patients with ERhighIL8low phenotype had significantly longer median RFS compared to patients with ERlowIL8high phenotype (150 and 114 months, respectively). This indicates that ER as a marker of favorable prognosis and IL8 as a marker of unfavorable prognosis (which is probably downregulated by ER), could influence the course of disease in an additive manner. It is interesting that according to our results, the impact of IL8 on survival is much stronger in PRlow tumours compared to PRhigh tumours (table 5). This 
Table 6. Survival analyses for subgroups of patients formed according to HER2 status and the median IL 8 level

\begin{tabular}{|c|c|c|c|c|c|c|}
\hline Phenotype & Number of patients & Number of relapses & Relapses (\%) & RFS (months) & Median RFS (months) & $\begin{array}{l}\text { p-Value } \\
\text { (Log Rank) }\end{array}$ \\
\hline HER2-IL8low & 27 & 4 & 14.8 & $19-161$ & 150 & \multirow{2}{*}{0.01} \\
\hline HER2-IL8high & 19 & 8 & 42.1 & $3-165$ & 64 & \\
\hline HER2+IL8low & 6 & 1 & 16.7 & $45-158$ & 153 & \multirow[t]{2}{*}{0.2} \\
\hline HER2+IL8high & 8 & 4 & 50 & $3-158$ & 100 & \\
\hline HER2-IL81ow & 27 & 4 & 14.8 & $19-161$ & 150 & \multirow[t]{2}{*}{0.9} \\
\hline HER2+IL8low & 6 & 1 & 16.7 & $45-158$ & 153 & \\
\hline HER2-IL8low & 27 & 4 & 14.8 & $19-161$ & 150 & \multirow[t]{2}{*}{0.02} \\
\hline HER2+IL8high & 8 & 4 & 50 & $3-158$ & 100 & \\
\hline HER2-IL8high & 19 & 8 & 42.1 & $3-165$ & 64 & \multirow[t]{2}{*}{0.2} \\
\hline HER2+IL 8low & 6 & 1 & 16.7 & $45-158$ & 153 & \\
\hline HER2-IL8high & 19 & 8 & 42.1 & $3-165$ & 64 & \multirow[t]{2}{*}{0.7} \\
\hline HER2+IL 8high & 8 & 4 & 50 & $3-158$ & 100 & \\
\hline
\end{tabular}

HER2-, HER2 gene not amplified; HER2+, HER2 gene amplified; IL81ow, IL8<88.82 pg/mg; IL8high, IL8 $\geq 88.82$ pg/mg.

could be explained by the fact that the most frequent subtype of tumours in this study was ERhighPRlow $(55 \%)$.

When patients were stratified according to HER2 status and the median IL8 level (table 6), IL8 expression had significant influence on survival of HER2-subgroup, but had no influence on survival of HER2+ subgroup. Patients with HER2-IL8low phenotype had significantly longer median RFS compared to patients with HER2-IL8high phenotype (150 and 64 months, respectively) and the number of relapses in HER2-IL8low subgroup was $15 \%$ versus $42 \%$ in HER2-IL8high subgroup. Although IL8 has been identified as a marker of HER2-enriched breast cancer, our results indicate the significant influence of IL8 on the course of disease of HER2-breast cancer. Furthermore, patients with HER2-IL8low phenotype had significantly longer median RFS compared to patients with HER2+IL8high phenotype (150 and 100 months, respectively). This implies that HER2 and IL8 as markers of unfavorable prognosis, could act additively on the course of disease.

According to our results, if IL8 is highly expressed, the influence of ER is weaker and that is the reason why there is no significant difference in RFS between patients with ERlowIL8high and ERhighIL8high phenotypes. The same is true for PR and HER2 and there is no significant difference in RFS between patients with PRlowIL8high and PRhighIL8high phenotypes, neither between patients with HER2-IL8high and HER2+IL8high phenotypes.

Literature related to the hormonal regulation of IL8 is controversial. Many authors found that multiple cytokines including IL8 were overexpressed in ER- breast cancer (Bièche et al. 2007; Chavey et al. 2007). On the other hand, Bendrik and Dabrosin found a significant positive correlation between IL8 and estradiol in normal breast tissue in situ and $\mathrm{ER}+\mathrm{PR}+$ breast cancer in vivo (Bendrik and Dabrosin 2009). Wen et al. first demonstrated in vitro and in mice in vivo that expression of IL8 was directly stimulated by activation of HER2 and downstream PI3K/Akt pathway (Wen et al. 2006). Azenshtein et al. showed that epidermal growth factor (HER2 ligand) potently upregulated IL8 secretion by breast tumour cells, and its effect was promoted by a consecutive treatment of the cells by estrogen and progesterone (Azenshtein et al. 2005). Haim et al. showed that EGF and estrogen upregulated in an additive manner the transcription and the secretion of IL8 in breast tumour cells (Haim et al. 2011). They postulated that EGF and estrogen probably act in independent transcriptional pathways that complement each other, in the upregulation of IL8.

Our results indicate significant contribution of IL8 on survival of hormonal dependent early-stage breast cancer patients and association with established parameters such as ER/PR and HER2. However, we must refrain and point that these results deserve further investigation, since we had small and unequal numbers of observations in subgroups. Our results are in contrast to the previously accepted hypothesis that IL8 expression is important feature that exclusively influences the course of ER- breast cancer. Furthermore, although IL8 has been identified as a marker of HER2-enriched breast cancer, our results indicate the significant influence of IL8 on the course of HER2-breast cancer. Moreover, not only expression of IL8 is dependent on ER and HER2 crosstalk, but also receptor-mediated signaling could act additively with IL8 in progression of hormone-dependent breast cancer. 
a

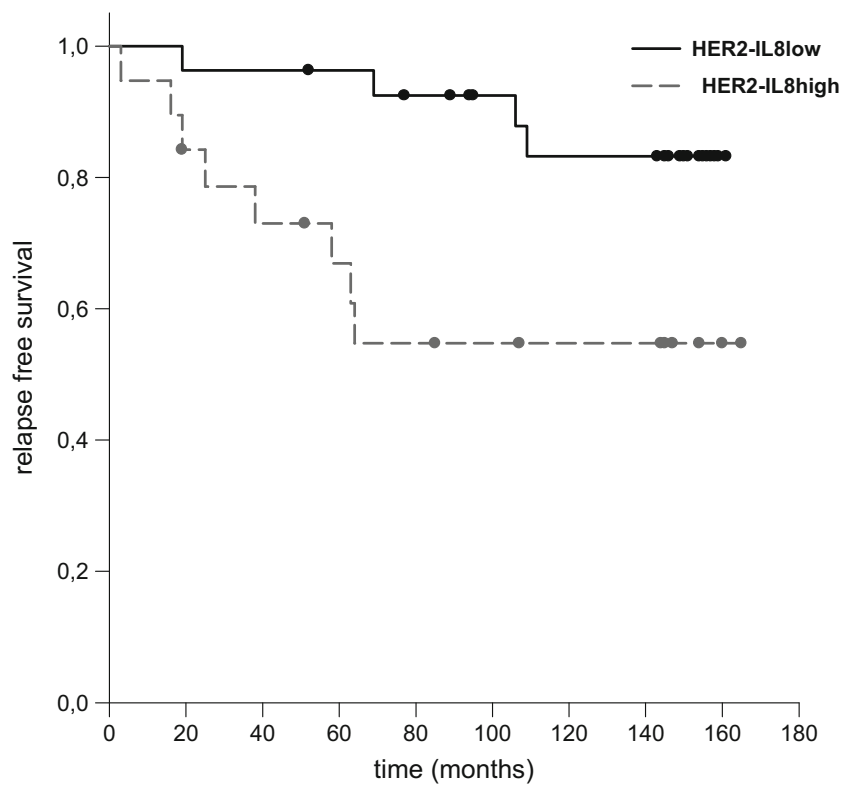

b

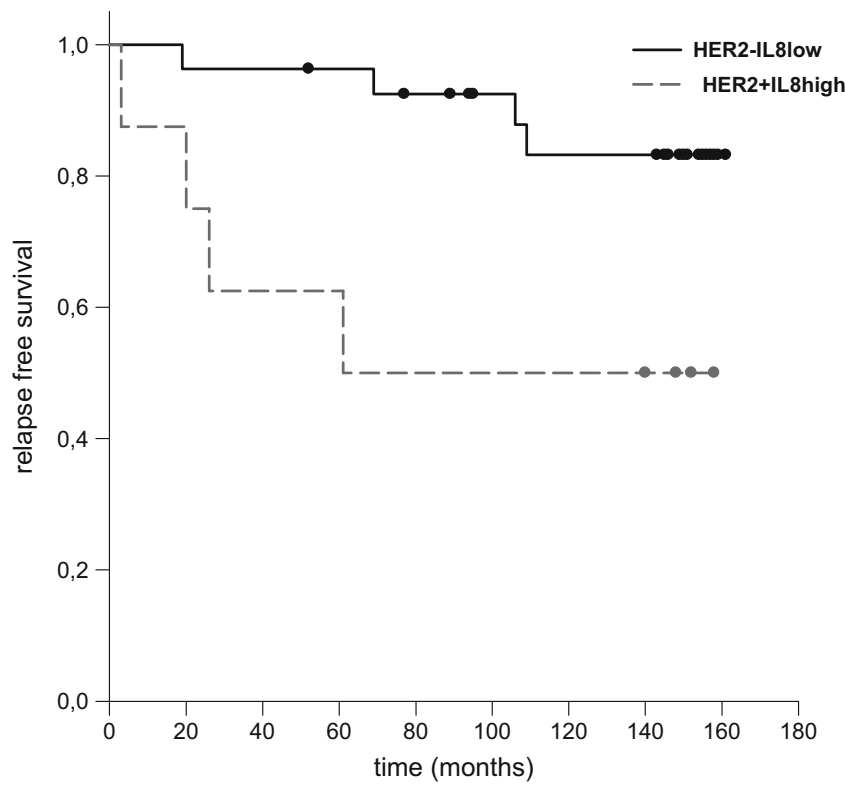

Figure 4. Survival analyses for subgroups of patients formed according to HER2 status and the median IL8 level (M=88.82 pg/ $\mathrm{mg})$.

\section{Acknowledgements}

This work was supported by Grant No.175068 'Molecular biomarkers of breast cancer and changes of their significance depending on the follow-up of the disease' from the Ministry of Science and Technological Development of the Republic of Serbia.

\section{References}

Abu Rabi Z, Todorović-Raković N, Vujasinović T, Milovanović J and Nikolić-Vukosavljević D 2015 Markers of progression and invasion in short term follow up of untreated breast cancer patients. Cancer Biomark. 15 745-754

Aceto N, Duss S, McDonald G, Meyer DS, Roloff TC, et al. 2012 Co-expression of HER2 and HER3 receptor tyrosine kinases enhances invasion of breast cells via stimulation of interleukin8 autocrine secretion. Breast Cancer Res. 14 R131

Azenshtein E, Meshel T, Shina S, Barak N, Keydar I and BenBaruch A 2005 The angiogenic factors CXCL8 and VEGF in breast cancer: regulation by an array of pro-malignancy factors. Cancer Lett. 217 73-86

Bendrik C and Dabrosin C 2009 Estradiol increases IL-8 secretion of normal human breast tissue and breast cancer in vivo. $J$. Immunol. 182 371-378

Benoy IH, Salgado R, Van Dam P, Geboers K, Van Marck E, et al. 2004 Increased serum interleukin- 8 in patients with early and metastatic breast cancer correlates with early dissemination and survival. Clin. Cancer Res. 10 7157-7162

Biasi F, Guina T, Maina M, Nano M, Falcone A, et al. 2012 Progressive increase of matrix metalloprotease- 9 and interleukin- 8 serum levels during carcinogenic process in human colorectal tract. PLoS One 7 e41839

Bièche I, Chavey C, Andrieu C, Busson M, Vacher S, et al. 2007 $\mathrm{CXC}$ chemokines located in the 4q21 region are up-regulated in breast cancer. Endocr. Relat. Cancer 14 1039-1052

Chavey C, Bibeau F, Gourgou-Bourgade S, Burlinchon S, Boissière $\mathrm{F}$, et al. 2007 Oestrogen receptor negative breast cancers exhibit high cytokine content. Breast Cancer Res. 9 R15

Chen Y, Shi M, Yu GZ, Qin XR, Jin G, et al. 2012 Interleukin-8, a promising predictor for prognosis of pancreatic cancer. World $J$. Gastroenterol. 18 1123-1129

Clark GM, McGuire WL, Hubay CA, Pearson OH and Marshall JS 1983 Progesterone receptors as a prognostic factor in Stage II breast cancer. N. Engl. J. Med. 309 1343-1347

Dunnwald LK, Rossing MA and Li CI 2007 Hormone receptor status, tumor characteristics, and prognosis: a prospective cohort of breast cancer patients. Breast Cancer Res. 9 R6

Freund A, Chauveau C, Brouillet JP, Lucas A, Lacroix M, et al. 2003 IL-8 expression and its possible relationship with estrogen receptor-negative status of breast cancer cells. Oncogene 22 256-265

Haim K, Weitzenfeld P, Meshel T and Ben-Baruch A 2011 Epidermal growth factor and estrogen act by independent pathways to additively promote the release of the angiogenic chemokine CXCL8 by breast tumor cells. Neoplasia 13 230-243

Kozłowski L, Zakrzewska I, Tokajuk P and Wojtukiewicz MZ 2003 Concentration of interleukin-6 (IL-6), interleukin-8 (IL-8) and interleukin-10 (IL-10) in blood serum of breast cancer patients. Rocz. Akad. Med. Bialymst. 48 82-84 
Li XJ, Peng LX, Shao JY, Lu WH, Zhang JX, et al. 2012 As an independent unfavorable prognostic factor, IL-8 promotes metastasis of nasopharyngeal carcinoma through induction of epithelial-mesenchymal transition and activation of AKT signaling. Carcinogenesis 33 1302-1309

Mannelqvist M, Stefansson IM, Bredholt G, Hellem BT, Oyan AM, et al. 2011 Gene expression patterns related to vascular invasion and aggressive features in endometrial cancer. Am. J. Pathol. 178 861-871

Milovanović J, Todorović-Raković N and Abu Rabi Z 2013 The prognostic role of interleukin-8 (IL-8) and matrix metalloproteinase -2 and -9 (MMP-2 and MMP-9) in node - negative untreated breast cancer patients. J. BUON. 18 866-873

Papaxoinis G, Pectasides DG, Korantzis I, Koutras A, Kosmidis PA, et al. 2010 Plasma angiogenic markers in patients with metastatic breast cancer treated with weekly docetaxel. $J$ Clin Oncol ASCO Annual Meeting Abstracts 28 e21004

Pine SR, Mechanic LE, Enewold L, Chaturvedi AK, Katki HA, et al. 2011 Increased levels of circulating interleukin 6, interleukin 8, C-reactive protein, and risk of lung cancer. J. Natl. Cancer Inst. 103 1112-1122

Qazi BS, Tang K and Qazi A 2011 Recent advances in underlying pathologies provide insight into interleukin-8 expression-mediated inflammation and angiogenesis. Int. J. Inflamm. 2011 908468

Reis ST, Leite KR, Piovesan LF, Pontes-Junior J, Viana NI, et al. 2012 Increased expression of MMP-9 and IL-8 are correlated with poor prognosis of bladder cancer. BMC Urol. 1218

Singh JK, Simões BM, Howell SJ, Farnie G and Clarke RB 2013 Recent advances reveal IL-8 signaling as a potential key to targeting breast cancer stem cells. Breast Cancer Res. 15210

Skoog L, Humla S, Axelsson M, Frost M, Norman A, et al. 1987 Estrogen receptor levels and survival of breast cancer patients. A study on patients participating in randomized trials of adjuvant therapy. Acta Oncol. 26 95-100

Slamon DJ, Clark GM, Wong SG, Levin WJ, Ullrich A and McGuire WL 1987 Human breast cancer: correlation of relapse and survival with amplification of the HER-2/neu oncogene. Science. 235 177-182

Snoussi K, Mahfoudh W, Bouaouina N, Fekih M, Khairi H, et al. 2010 Combined effects of IL-8 and CXCR2 gene polymorphisms on breast cancer susceptibility and aggressiveness. BMC Cancer 10283

Todorović-Raković N and Milovanović J 2013 Interleukin-8 in breast cancer progression. J. Interferon Cytokine Res. 33 563570

Wang Y, Xu RC, Zhang XL, Niu XL, Qu Y, et al. 2012 Interleukin8 secretion by ovarian cancer cells increases anchorageindependent growth, proliferation, angiogenic potential, adhesion and invasion. Cytokine 59 145-155

Wen XF, Yang G, Mao W, Thornton A, Liu J, et al. 2006 HER2 signaling modulates the equilibrium between pro- and antiangiogenic factors via distinct pathways: implications for HER2targeted antibody therapy. Oncogene 25 6986-6996

Xie K 2001 Interleukin 8 and human cancer biology. Cytokine Growth Factor Rev. 12 375-391

Yao C, Lin Y, Chua MS, Ye CS, Bi J, et al. 2007 Interleukin8 modulates growth and invasiveness of estrogen receptornegative breast cancer cells. Int. J. Cancer 121 1949-1957

MS received 29 September 2016; accepted 13 February 2017

Corresponding editor: RitA MULHERKAR 